\title{
Hilson index and renal transplant rejection: a single-center experience
}

This article was published in the following Dove Press journal:

Research and Reports in Nuclear Medicine

27 March 2013

Number of times this article has been viewed

\section{Muhammad Choudhry Nitesh Rao \\ Department of Renal Medicine, Royal Adelaide Hospital, Adelaide, SA, Australia}

Correspondence: Muhammad Choudhry Department of Renal Medicine, Royal Adelaide Hospital, Adelaide, SA, Australia

Tel +6I 261747032

Email MuhammaderfanChoudhry@act. health.gov.au
Background: The Hilson index has been used in addition to sequential images and changes in posttransplant region-of-interest curves to separate rejections from acute tubular necrosis. The aim of the present retrospective study was to evaluate the role of the Hilson index in accurately diagnosing renal transplant rejection and to explore the correlation between changes in the perfusion index across serial scans with the incidence of rejection.

Methods: Using the Hilson index, we retrospectively examined 150 renal biopsies and 250 perfusion scans from 150 renal transplant recipients from 2010 to 2012 at our center. A Hilson index above 150 was labeled as positive and anything less as negative. Renal transplant rejection was diagnosed by correlating clinical suspicion with the histological findings of the renal biopsy. The Hilson index closest to the perfusion scan and the histology findings on renal biopsy were used to calculate the sensitivity and specificity of the index in diagnosing renal transplant rejection. Logistical regression was used to determine the relationship between absolute and percentage changes in perfusion scan indices with histological findings.

Results: The Hilson index had a sensitivity of $26 \%$ and a specificity of $82 \%$ in predicting renal transplant rejection. The Hilson index was not specific in differentiating between acute tubular necrosis and renal rejection. The absolute and percentage changes in value of the index did not correlate with renal transplant rejection.

Conclusion: At our center, the Hilson index had poor sensitivity for diagnosing renal transplant rejection. The absolute and percentage changes in the Hilson index across serial scans failed to correlate with renal transplant rejection. The findings of this audit suggest the need for individual clinical discretion and the use of the Hilson index as supportive information only. This has raised interest in utilizing resistive indices from renal ultrasound for this purpose at our center. The sensitivity and specificity of Doppler ultrasound have been inconsistent and observer-dependent in various studies. Magnetic resonance imaging has been proposed but is unrealistic. Due to the nature of the test and its results, an audit cycle was not considered plausible.

Keywords: Hilson index, renal transplant rejection, acute tubular necrosis

\section{Introduction}

In the postoperative management of renal transplantation, there is a need for a noninvasive method of diagnosing vascular insufficiency, acute tubular necrosis, rejection, and problems of urinary outflow tract obstruction. An effective method should be safe, rapid, and simple, and should be repeatable at intervals of 48-72 hours during the critical 2- to 3-week period following surgery. To date, the most satisfactory method to use is the renal nuclear scan.

Damage to renal cortical microcirculation, an early event in the course of acute rejection crisis, usually precedes measurable functional derangements in the submit your manuscript | www.dovepress.com

Dovepress

http://dx.doi.org/10.2147/RRNM.S38274
Research and Reports in Nuclear Medicine 2013:3 I-7

(C) 2013 Choudhry and Rao, publisher and licensee Dove Medical Press Ltd. This is an Open Access article which permits unrestricted noncommercial use, provided the original work is properly cited. 
transplanted kidney. Direct assessment of renal blood flow by renal nuclear scan may provide a sensitive and reliable index to the diagnosis of acute rejection, with particular regard to the differential diagnosis of acute rejection and acute tubular necrosis. ${ }^{1,2}$ The perfusion index is a standard method for quantitative evaluation of renal transplant perfusion, with the Hilson index being one popular example. The Hilson index, utilizing Tc-99m diethylene triamine pentaacetic acid (DTPA), was first used in 1978 in 955 perfusion scans on 152 patients with 167 renal transplants. Regions of interest (ROIs) are defined over the iliac artery just distal to the transplant, the transplant, and a background area lateral to the kidney. Normalized time-activity curves are generated from these ROIs. The index is calculated by dividing the area under the arterial curve by the peak area under renal curve times 100 .

Given the paucity of quality evidence, we undertook an audit to determine the adequacy of the Hilson index for the prediction of renal transplant rejection in the immediate posttransplant period. The other objective was to explore the relationship between changes in the Hilson index on serial scans and its correlation with renal transplant rejection. The perceptual trigger for the audit was the high volume (120 scans per year) and costs of renal perfusion scans (A\$332 each) conducted at our unit in the immediate posttransplant period to differentiate between acute tubular necrosis and rejection.

A PubMed search with two keywords and personal contact with one expert in the subject found no previous studies since its inception in 1978 on the accuracy of the Hilson index in predicting renal transplant rejection. Only one prospective study has looked at the ability of renal perfusion scan to predict renal transplant rejection, but it used the cortical perfusion index and had only 15 patients. ${ }^{1}$ We therefore conducted a retrospective study extending over 2 years to determine the ability of the Hilson index to accurately predict renal transplant rejection.

There is wide variation in practice across various transplant units, with some institutions using the Hilson index posttransplant, while others use resistive indices from a renal ultrasound in preference to the perfusion scan. The Royal Adelaide Hospital, Adelaide, SA, Australia renal transplant unit performs 80 transplants per year on average. The management protocol mandates radionuclide scans in the immediate postoperative period for all renal transplant recipients and on alternate days for delayed graft function. In suspected graft rejection, an increase in the Hilson index serves to guide management in terms of lowering the threshold for a renal biopsy in our unit.

\section{Materials and methods}

\section{Study design}

This was a retrospective study in the form of an audit of renal transplants performed at the Royal Adelaide Hospital between 2010 and 2012. Data were collected from CNARTS, ANZDATA, and patients' case notes. The inclusion criteria were: (1) renal transplants performed between 2010 and 2012; (2) renal biopsies performed posttransplant; and (3) renal nuclear scans performed posttransplant with estimation from the Hilson index. Patients were excluded who had perfusion scans using different indices, or who had no nuclear scans.

\section{Study protocol}

A single biopsy was performed on all included patients. A renal perfusion scan was performed within 24 hours posttransplant for all patients. Patients with delayed graft function underwent renal perfusion scans on alternate days. Delayed graft function was defined as a drop in serum creatinine of less than $10 \%$ every 24 hours or requiring dialysis within 5 days of transplant at our center.

Where more than one perfusion scan had been performed following biopsy, only the scans nearest to the date of biopsy were considered for this study. The biopsies were performed using a Tru-Cut biopsy needle. The samples were processed using standard techniques for light microscopy, electron microscopy, and immunofluorescence studies. All biopsy slides were examined by a pathologist who had no knowledge of the renal scan results. Rejection was defined retrospectively as patients who were given treatment for rejection, dictated by biopsy findings and individual clinical discretion. There are no standard guidelines available, but a significant rise in the Hilson index, particularly when above a threshold of 150 , has been considered suggestive of rejection in patients with delayed graft function at our unit. No ethical issues were identified in undertaking this audit.

\section{Imaging}

During imaging, the patients lay supine on their beds, with the head of the gamma camera positioned over the transplant site, so that the bladder and iliac artery were in the field of view. A 300-400 MBq MAG3 was injected into a peripheral vein, shunt, or fistula. The first image (vascular phase) was recorded for 30 seconds, starting at the time of tourniquet release. The next image ( 2 minutes) was recorded for 300,000 counts on a standard-field camera, or for 400,000 counts on a wide-field camera. The time required for this image-taking 
was noted, and subsequent images were recorded for the same duration at 30 minutes after injection. The data were recorded using a dedicated minicomputer with a $64 \times 64$ matrix.

\section{Quantitation}

ROIs were defined over the iliac artery just distal to the transplant (usually best seen between 6 and $20 \mathrm{sec}$ after injection), over the transplant (being careful not to include any portion of the kidney that overlies the iliac artery), and over a background area lateral to the kidney. Normalized time-activity curves were generated from these ROIs. These were displayed using frame numbers rather than time for the abscissa, which has the effect of expanding the initial (vascular) portion of the curves.

A computer program then calculated the areas under the normalized arterial and renal curves up to the time of the peak of the arterial curve caused by the first passage of the bolus, with the peak being selected by the operator. The computer printed out the ratio of these areas as an index, given by dividing the area under the arterial curve by the peak area under renal curve multiplied by 100 . The index has a value of 100 where two areas are identical. As relative blood flow through the kidney falls, the area under the vascular phase of the renal curve becomes smaller, ${ }^{3}$ increasing the index. Conversely, as renal blood flow improves, the index falls.

The permanent record of the study consists of the images, the printout with the perfusion index, photographic records of the ROIs, the time activity curves, and the backgroundcorrected renal curve.

\section{Statistical methods}

The relationship between absolute and percentage changes in the perfusion index with renal transplant rejection was analyzed by logistic regression.

The statistical power of an analysis is the likelihood that a false null hypothesis will be rejected under specified assumptions concerning the size of the effect for the independent variable, the sample size, and the significance level for rejecting the null hypothesis. In order to determine the statistical power of the design, we used the $\mathrm{G}^{*}$ Power 3 software package (Heinrich Heine Universität Düsseldorf, Dusseldorf, Germany), basing the calculation of power on the assumption that the null hypothesis would be rejected at the 0.05 significance level. That assessment is based on the assumption that, under the alternate hypothesis, the probability of rejection is twice the probability that would apply under the null hypothesis (ie, a strong effect). For the detection of strong effects, the sample size of 65 cases provides adequate power $(>0.95)$. For a study of diagnostic indicators, it is reasonable to expect that the predictor should have strong statistical effects

\section{Results}

We examined 150 renal biopsies from 150 transplant recipients. A single biopsy was performed for all included patients. Patients with delayed graft function underwent renal perfusion scans on alternate days. A total of 250 perfusion scans were performed. The patients were categorized into two groups, using renal biopsy as their gold standard: normal and acute tubular necrosis (ATN) (102) and acute rejection (48). The renal perfusion scans were reported as normal (51), ATN (90), and rejection/ATN (8).

Sixty-five patients had delayed graft function. Of these, the number of patients with two, three, and four perfusion scans was 30, 26, and 9, respectively. There were no deaths among the patient population.

\section{Sensitivity and specificity}

Patients with a Hilson index of more than 150 were labeled as positive and less than 150 as negative. Patients were divided into four groups: (1) "true negative" was defined as a Hilson index of less than 150 and no rejection on renal biopsy; (2) "true positive" was defined as a Hilson index of more than 150 and rejection on renal biopsy; (3) "false positive" was defined as a Hilson index of more than 150 and no rejection on biopsy; and (4) "false negative" was defined as a Hilson index of less than 150 and rejection on renal biopsy. The tabular forms of these results, as well as the calculations of sensitivity and specificity, are presented in Tables 1 and 2, respectively.

\section{Statistical analysis}

Relationship between absolute changes in the perfusion index and renal transplant rejection

The logistic regression analysis found that the relationship between the absolute change in the renal perfusion index and rejection was not statistically significant. The coefficient for change in the perfusion index was -0.001 (Wald $=0.030$, $\mathrm{df}=1.000, P=0.861)$. The null hypothesis states that the likelihood of rejection is not associated with changes in the perfusion index (ie, beta $=0$ ). The results indicate that the null hypothesis should be retained.

Table I Test outcomes by transplant results

\begin{tabular}{lll}
\hline & \multicolumn{2}{l}{ Transplant result } \\
\cline { 2 - 3 } & Rejected & Not rejected \\
\hline Test outcome & & \\
Positive & $\mathrm{TP}=1 \mathrm{I}$ & $\mathrm{FP}=19$ \\
Negative & $\mathrm{FN}=30$ & $\mathrm{TN}=90$ \\
\hline
\end{tabular}


Figure 1 shows the relationship between rejection on the $\mathrm{x}$-axis $(0=$ not rejected; $1=$ rejected $)$ and absolute changes in the perfusion index on the $y$-axis. Logistic regression analyses revealed that the likelihood of rejection was not associated with absolute changes in the perfusion index.

\section{Relationship between percentage changes in the} perfusion index and renal transplant rejection

The logistic regression analysis found that the relationship between percentage changes in the renal perfusion index and rejection was not statistically significant. The coefficient for percentage changes in the perfusion index was -0.004 (Wald $=0.638$, df $=1.000, P=0.424$ ). The null hypothesis states that the likelihood of rejection is not associated with change in the perfusion index (ie, beta $=0$ ). The results indicate that the null hypothesis should be retained.

\section{Discussion}

The Hilson index has been used in addition to sequential images and changes in the ROI curves to separate rejection from ATN. ${ }^{3}$ To our knowledge, this is the first retrospective study investigating the efficacy of the Hilson index in this regard since its inception in 1978. However, there was one study that analyzed the adequacy of cortical perfusion index correlating with renal biopsy findings. This was a prospective study using DTPA with 34 patients. The results showed a sensitivity of $63 \%$ and specificity of $80 \%{ }^{2}$

Our audit showed a sensitivity of $26 \%$ and specificity of $82 \%$ (Table 2). It is generally agreed that studies of renal blood flow do not adequately reflect the complexity of the microcirculation of the kidney with its inherent autoregulation and redistribution of blood from the cortex. ${ }^{2}$ As the global renal perfusion index measures changes in blood flow in both compartments, the marked decrease in cortical perfusion may be masked by the relative preservation of medullary flow, which is relatively unaffected early in the course of acute rejection crisis. These data may shed light on the poor sensitivity of the renal perfusion index in predicting acute rejection, as noted in our study in comparison with the prospective study mentioned above. However, the renal scan is not interpreted solely according to the index, which simply serves as a guide.

Table 2 Results

\begin{tabular}{llll}
\hline Index & Formula & Calculation & Result \\
\hline Sensitivity & $(\mathrm{TP} / \mathrm{TP}+\mathrm{FN}) \times 100$ & $(\mathrm{II} / \mathrm{II}+30) \times 100$ & $26.8 \%$ \\
Specificity & $(\mathrm{TN} / \mathrm{TN}+\mathrm{FP}) \times 100$ & $(90 / 90+19) \times 100$ & $82.6 \%$ \\
\hline
\end{tabular}

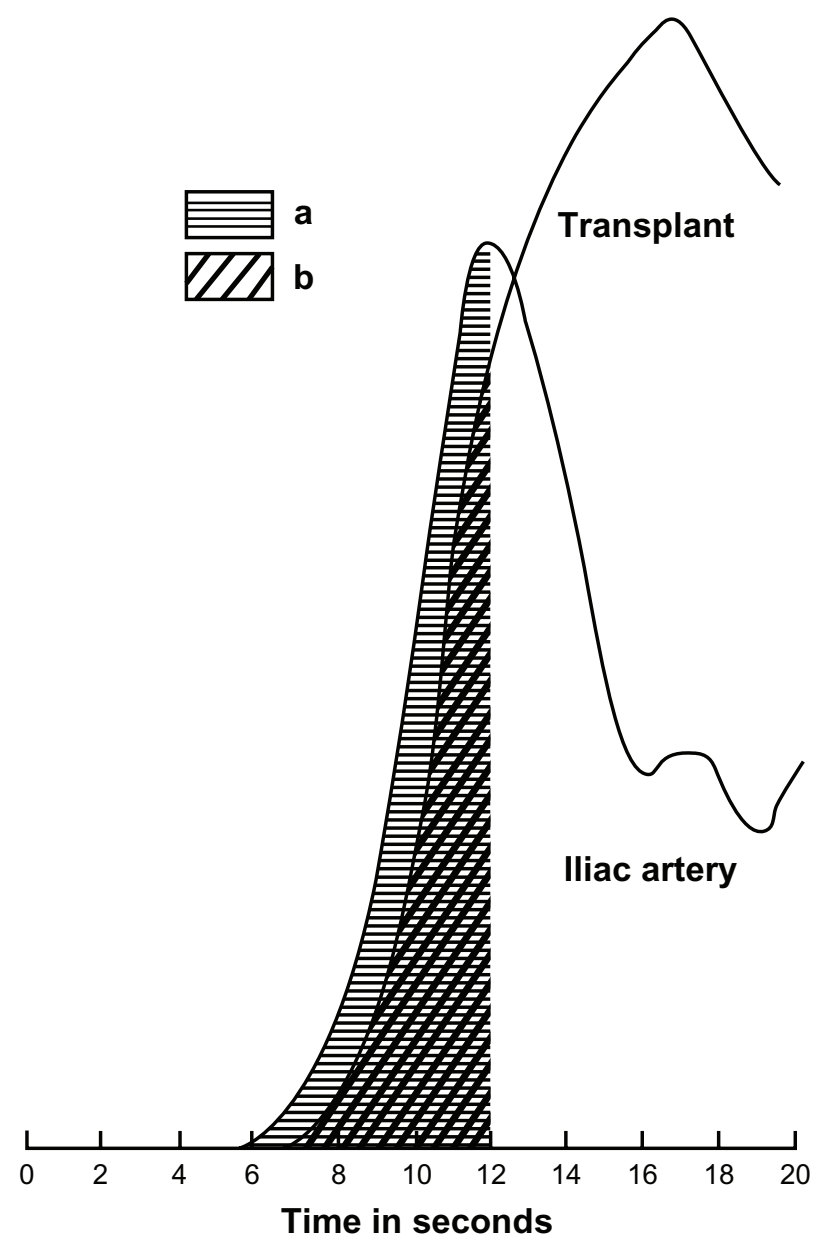

Figure I Areas used for perfusion index, given by $(\mathrm{a} / \mathrm{b}) \times 100$.

It has been 26 years since the Hilson index was tested. A general switch from $25 \mathrm{~cm}$ field-of-view cameras to $40 \mathrm{~cm}$ field-of-view cameras has been made. TC MAG3 has started replacing DTPA ${ }^{4}$ because of better image resolution, but it is more expensive. Technetium-99 MAG3 is cleared primarily by proximal tubules with minimal filtration and an extraction fraction of $50 \%$. Its clearance can be used to measure effective renal plasma flow. ${ }^{5}$ These changes may also account for the different results obtained at our center in comparison with those obtained by Hilson et al. ${ }^{3}$

An aspect of the technique that has been overlooked is that there are no absolutes - what matters is the change in perfusion and function, with each patient acting as his own control. ${ }^{4}$ The results of our study showed that the absolute changes in value of perfusion scans are not correlated with renal transplant rejection (Figure 2 and Table 3). We also investigated the relationship between percentage changes in the Hilson index over serial scans and renal transplant rejection. We failed to detect any significant correlation (Table 4). 


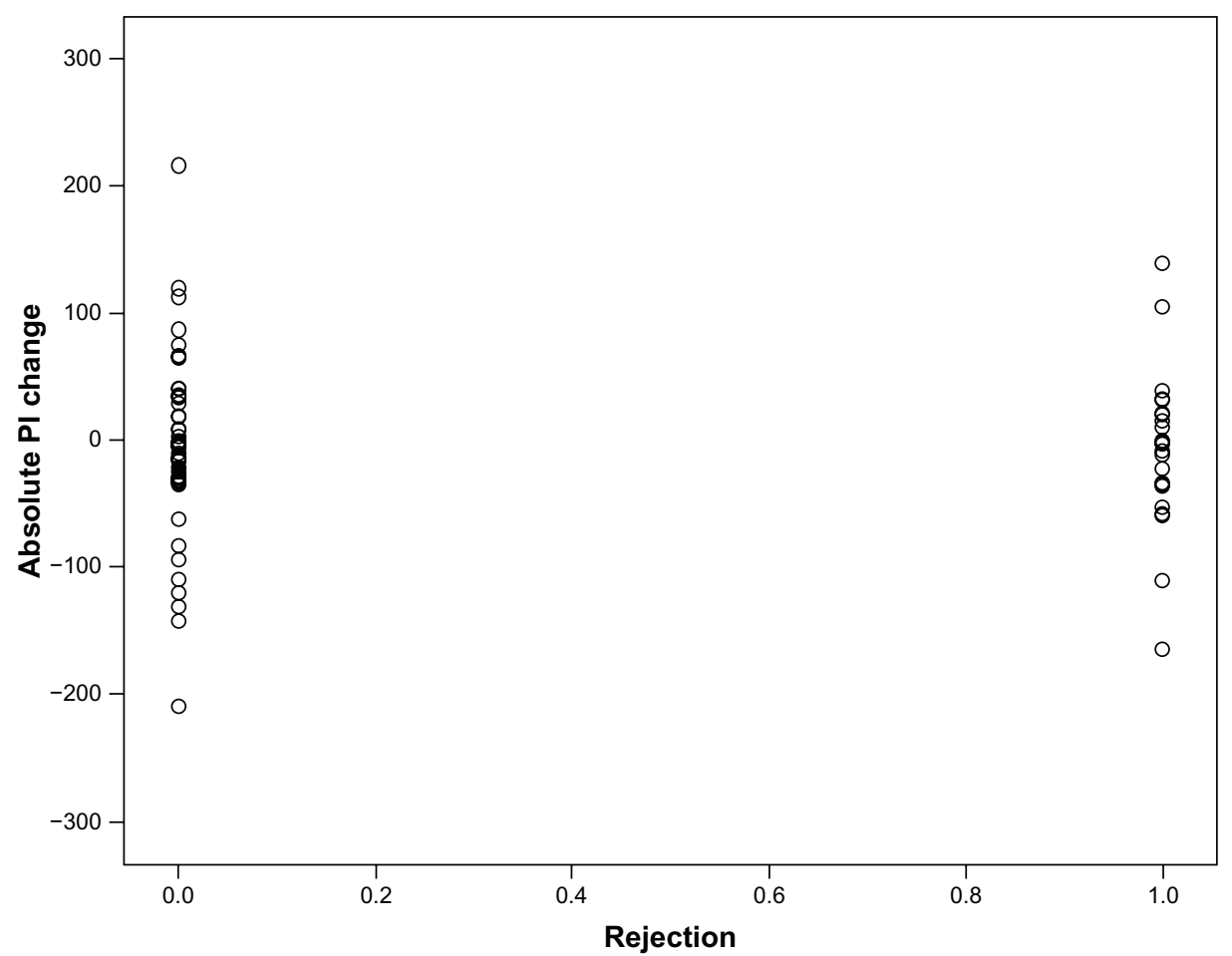

Figure 2 Absolute change in perfusion index $(\mathrm{PI})$ and rejection.

Some degree of tubular necrosis after renal transplantation has been reported in as many as $50 \%$ of recipients of cadaveric donor kidneys. ${ }^{6}$ It has been reported that the renal scan can accurately differentiate between renal allograft rejection and acute tubular necrosis, especially with serial studies. ${ }^{7}$ However, this has not been consistent, as it cannot be assumed that the initial scan will demonstrate the maximum perfusion and the functional aberration associated with acute tubular necrosis, nor that the cortical perfusion will be normal in transplanted kidneys with acute tubular necrosis.

The index is not specific in differentiating between rejection and ATN. One study retrospectively looked at 15 patients with a clinical diagnosis of either acute rejection or ATN. Technetium scan diagnosis did not agree with the clinical assessment in nine of these patients. ${ }^{8}$ Our audit agrees with these observations, as two-thirds of the patients with a raised index had ATN rather than rejection.

The use of 99MTc sulfur colloid and fibrinogen deposition in the rejecting kidney have shown promise with regard to diagnosing rejection, ${ }^{9,10}$ but, despite increasingly

Table 3 Variable(s) entered: absolute change

\begin{tabular}{lllllll}
\hline & B & SE & Wald & df & Sig & Exp(B) \\
\hline Delta & -0.001 & 0.004 & 0.030 & 1.000 & 0.861 & 0.999 \\
Constant & -0.772 & 0.273 & 7.970 & 1.000 & 0.005 & 0.462 \\
\hline
\end{tabular}

sophisticated analysis of radiopharmaceutical kinetics, a radionuclide quantitative method to distinguish ATN from rejection with a consistent degree of specificity has not yet been developed. A decline in diastolic blood flow velocity is a sensitive sign of increased renovascular resistance on Doppler ultrasound, ${ }^{11}$ but the role of ultrasound resistive indices (RI) in diagnosing renal transplant rejection is uncertain, with a sensitivity and specificity of $79 \%$ and $90 \%$, respectively, ${ }^{12}$ to only $35 \%$ sensitivity and specificity. ${ }^{13}$ This may be due to ultrasound being operator-dependent and the fact that, in certain cases in which the diastolic blood flow velocity is significantly decreased, the calculation of a valid RI is hindered. ${ }^{14}$

Other confounding factors include the fact that transplanted kidneys have significantly higher intrarenal arterial flow indices in comparison with native kidneys. RI and pulsatality indices (PI) increase with age in both the native kidneys and the allograft. However, the increase in the transplanted kidney correlates with the age of the recipient but not with the age of the donor. Corresponding to the increase in RI and PI, the

Table 4 Variable(s) entered: percent change

\begin{tabular}{lllllll}
\hline & B & SE & Wald & df & Sig & $\operatorname{Exp(B)}$ \\
\hline Change (\%) & -0.004 & 0.005 & 0.638 & 1.000 & 0.424 & 0.996 \\
Constant & -0.737 & 0.273 & 7.275 & 1.000 & 0.007 & 0.479 \\
\hline
\end{tabular}


blood pressure is significantly elevated in the elderly. ${ }^{13}$ The degree of external pressure with the transducer on the graft also has an impact on the intrarenal arterial Doppler findings and measurements obtained. ${ }^{13}$ Distinct interstitial infiltrations, edemas of any origin, vascular changes, and chronic rejection and arteriosclerosis also lead to elevated RI. ${ }^{15}$

PI, which incorporate mean blood flow velocity over a cardiac cycle, may be a more accurate and useful measure of overall transplant perfusion. The serial investigation of PI allows better recognition of rejection episodes than a single measurement of RI or PI. ${ }^{13}$

Improvement in the perfusion index could be achieved by using the cortical perfusion index instead of global perfusion. $^{2}$

In the calculation of perfusion indices, correction for administered activity is often accomplished using activity measurements made over a convenient artery, which, therefore, has a critical influence on the value of the index obtained. The errors in repeat studies on the same patient may be as high as $39 \%$ and between patients as high as $53 \%$. These figures can be reduced to below $18 \%$ and $21 \%$, respectively, by constructing an ROI to extend over as much of the arterial width as possible, rather than relying only on the maximum pixel count. Further reduction to below $12 \%$ and $10 \%$ is possible by utilizing a 128 $\times 128$ acquisition matrix instead of $64 \times 64$, and by drawing the ROI over the aorta instead of the iliac artery. ${ }^{16}$

It has been suggested that the technique can be improved by estimating the noninvasive measurement of renal blood flow based on the principle of fractionation of cardiac output using any recirculating gamma-emitting tracer, but this is difficult to implement practically. ${ }^{17}$

A major limitation of our study is the lack of information regarding the blood pressure, cardiac output, and quality of the bolus during the perfusion scan for the patient population. Another weakness in this audit is the absence of comparisons between demographic characteristics, the quality of donor kidneys, HLA status in the two patient groups with and without rejection, and its influence on the results of the audit.

\section{Conclusion}

We conclude that the best practice guidelines as a result of this audit for our center are as follows:

- The Hilson index has poor sensitivity for diagnosing renal transplant rejection.

- The Hilson index has poor specificity for differentiating between renal transplant rejection and acute tubular necrosis.
- The trend in Hilson index over sequential imaging and individual clinical discretion is the current policy in the renal transplant unit at the Royal Adelaide Hospital. As shown in the analysis above, the change in perfusion index, both in terms of absolute value and percentage changes, failed to be predictive of renal rejection.

\section{Disclosure}

The authors report no conflicts of interest in this work.

\section{References}

1. Anaise D, Oster ZH, Atkins HL, et al. Cortex perfusion index: a sensitive detector of acute rejection crisis in transplanted kidneys. J Nucl Med. 1986;27:1697-1701.

2. Tourville DR, Kim DU, Viscuso R, Jacobs M, Schoen S, Fillipone D. Anticipation of renal transplant failure by postanastomosis biopsy and immunofluorescence. Transplant Pros. 1977;9:91-95.

3. Hilson AJ, Maisey MN, Brown CB, Ogg CS, Bewick MS. Dynamic renal transplant imaging with TC-99m DTPA supplemented by a transplant perfusion index in management of renal transplants. $J$ Nucl Med. 1978;19:994-1000.

4. Hilson AJ. The renal transplant perfusion index. Where are we now? Eur J Nucl Med. 1991;18:227-228.

5. O'Malley JP, Ziessman HA, Chantarapitak N. Tc-99m MAG3 as an alternative to Tc-99m DTPA and I-131 Hippuran for renal transplant evaluation. Clin Nucl Med. 1993;18:22-29.

6. Cho SI, Olsson CA, Bradley JW, Nasbeth DC. Regional program for kidney preservation and transplantation in New England. Am J Surg. 1976;131:428-433.

7. Mandel SR, Mattern WD, Staab E, Johnson G Jr. Use of radionuclide imaging in the early diagnosis and treatment of renal allograft rejection. Ann Surg. 1975;181:596-603.

8. Delmonico FL, McKusick KA, Cosimi AB, Russell PS. Differentiation between renal allograft rejection and acute tubular necrosis by renal scan. AJR Am J Roentgenol. 1977;128(4):625-628.

9. Frick MP, Loken MK, Goldberg ME, Simmons RL. Use of $99 \mathrm{~m} \mathrm{Tc}$ sulfur colloid in evaluation of renal transplant complications. $J$ Nucl Med. 1976;17:181-183.

10. George EA, Codd JE, Newton WT, Haibach H, Donati RM. Comparative evaluation of renal transplant rejection with radionuclide fibrinogen, 99M Tc-sulfur colloid and 67 Ga-citrate. J Nucl Med. 1976;17:175-180.

11. Norris CS, Barnes RW. Renal artery flow velocity analysis: a sensitive measure of experimental and clinical renovascular resistance. $J$ Surg Res. 1984;36:230-236.

12. Rigsby CM, Burns PN, Weltin GG, Chen B, Bia M, Taylor KJ. Doppler signal quantitation in renal allografts: comparison in normal and rejecting transplants, with pathologic correlation. Radiology. 1987; 162(1 Pt 1):39-42.

13. Mallek R, Mostbeck G, Kain R, et al. [Vascular kidney transplant rejection - is a duplex sonographic diagnosis possible?] Rofo. 1990;152(3):283-286. [German].

14. Genkins SM, Sanfilippo FP, Carroll BA. Duplex Doppler sonography of renal transplants: lack of sensitivity and specificity in establishing pathologic diagnosis. AJR Am J Roentgenol. 1989;152(3):535-539.

15. Flückiger F, Steiner H, Horn S, Ratschek M, Deu E. [Color-coded duplex sonography and the resistive index in dysfunctional kidney transplants]. Rofo. 1990;153(6):692-697. German.

16. Hamilton D, Miola UJ, Payne MC. The renal transplant perfusion index: reduction in the error and variability. Eur J Nucl Med. 1994;21:232-238.

17. Peters AM, Gunasekera RD, Henderson BL, et al. Noninvasive measurement of blood flow and extraction fraction. Nucl Med Commun. 1987;8(10):823-837. 
Research and Reports in Nuclear Medicine

Dovepress

\section{Publish your work in this journal}

Research and Reports in Nuclear Medicine is an international, peerreviewed, open access journal publishing original research, reports, reviews and commentaries on all areas of nuclear medicine. The manuscript management system is completely online and includes a very quick and fair peer-review system. Visit http://www.dovepress.com/ testimonials.php to read real quotes from published authors.

Submit your manuscript here: http://www.dovepress.com/research-and-reports-in-nuclear-medicine-journal 